\title{
La tradición obstétrica familiar en el Real Colegio de Cirugía de San Carlos de Madrid: Concepción de Navas, la hija de Juan de Navas
}

The obstetric family tradition at the Real Colegio de Cirugía de San Carlos de Madrid: Concepción de Navas, the daughter of Juan de Navas

\section{A tradição obstétrica famíliar no Real Colegio de Cirugía de San Carlos de Madrid: Concepción de Navas, filha de Juan de Navas}

\author{
María Dolores Ruiz-Berdún²
}

\begin{abstract}
${ }^{1}$ Matrona, Diplomada en Estudios Avanzados en Historia de la Ciencia por la Universidad de Alcalá, profesora de la Unidad Docente de Matronas de Madrid, profesora colaboradora de la EUE Cruz Roja de Madrid

Cómo citar este artículo en edición digital: Ruiz Berdun, $M^{a}$. D. (2012) La tradición obstétrica familiar en el Real Colegio de Cirugía de San Carlos de Madrid: Concepción de Navas, la hija de Juan de Navas. Cultura de los Cuidados. (Edición digital) 16, 32. Disponible en: $h t t p: / / d x$. doi.org/10.7184/cuid.2012.32.09

Correspondencia: C/ García Morato nº 48. 28805 Alcalá de Henares (Madrid).ruizberdun@gmail.com (605-66-88-07) Recibido: 06/12/2011 - Aceptado: 11/02/2012
\end{abstract}

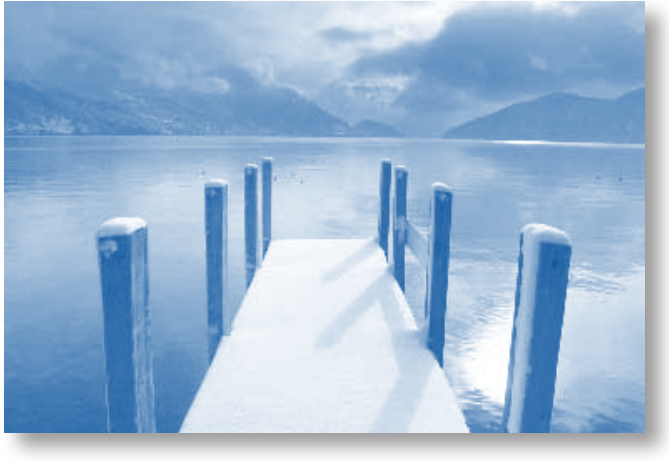

ABSTRACT

This article basically, studied the documentation which is preserved in the archive of the Universidad Complutense de Madrid and the Archivo Histórico Nacional concerning to the family Navas. Juan de Navas was an eminent surgeon of end of century XVIII, author of Elementos del arte de partear, ending his professional career as Vice - Director of the Real Colegio de Cirugía de San Carlos de Madrid. In this centre, a few years later, his daughter Concepción de Navas studied midwifery. This research delves into the figure of these two health professionals and shows the harsh conditions of life for the professional practice existing in Madrid in the 18 th and 19th centuries.

Keywords: midwives, education, Colegio de Cirugía de San Carlos, Juan de Navas, Concepción de Navas.

\section{RESUMO}

Este artigo basicamente, estudos a documentação que é preservada no arquivo da Universidad Complutense de Madrid e a Archivo Histórico Nacional relacionados com o família Navas. Juan de Navas foi um eminente cirurgião do final do século XVIII, autor do libro Elementos del arte de partear, terminou a sua carreira profissional como Vice-Director do Real Colegio de Cirugía de San Carlos, centro que, anos mais tarde, sua filha Concepción de Navas estudou da parteira. Com esta pesquisa se aprofunda na figura desses dois profissionais de saúde e delineando as duras condições de vida para a prática profissional existente em Madrid nos séculos XVIII e XIX. 
Palavras-chave: parteiras, formação, Colegio de Cirugía de San Carlos, Juan de Navas, Concepción de Navas.

\section{RESUMEN}

En el presente artículo se estudia, básicamente, la documentación que se conserva en el Archivo General de la Universidad Complutense de Madrid y en el Archivo Histórico Nacional relativa a la familia Navas. Juan de Navas fue un eminente cirujano de finales del siglo XVIII, autor de unos Elementos del arte de partear, que terminó su carrera profesional siendo vice-director del Real Colegio de Cirugía de San Carlos de Madrid, centro en el que, años más tarde, su hija Concepción de Navas realizó los estudios de matrona. Con la presente investigación se profundiza en la figura de estos dos profesionales sanitarios y se exponen las duras condiciones de vida que para el ejercicio profesional existían en Madrid en los siglos XVIII y XIX.

Palabras clave: matronas, formación, Colegio de Cirugía de San Carlos, Juan de Navas, Concepción de Navas.

\section{INTRODUCCIÓN}

El Real Colegio de Cirugía de San Carlos de Madrid inició su andadura como formador de cirujanos civiles el uno de octubre de 1787 (Usandizaga, 1948). Entre los ocho profesores designados inicialmente para ocupar las Cátedras del Colegio se encontraba Juan de Navas (Aparicio, 1956).

Juan de Navas Ramos era natural de Archidona (Málaga), y debió nacer antes de 1749, ingresando en el Real Colegio de Cirugía de la Armada de Cádiz en 1765, donde posteriormente fue profesor (Herrera, 1989).
El 19 de abril de 1790 se inauguró la Cátedra de Partos en el Real Colegio de Cirugía de San Carlos de Madrid (Burke, 1977). Según se recogía en la Ordenanzas del Colegio de 1787, dentro de las funciones del catedrático se encontraba el formar a todas aquellas mujeres que quisieran aprender el «arte de partear». A esta enseñanza, que debía hacerse en una de las salas del Colegio «a puertas cerradas», sólo podían concurrir aquellas mujeres que fuesen casadas, circunstancia que debía ser acreditada con un certificado de matrimonio. También debían adjuntar una autorización del marido para poder asistir a instruirse como futuras matronas. El Catedrático de partos en 1790 era Agustín Ginesta, que había ganado la plaza tras quedar vacante por la muerte de Jaime Respau el 31 de julio de 1788 (Aparicio, 1956).

La enseñanza de las matronas en los Colegios de Cirugía, supuso a la larga la pérdida de su autonomía profesional debido al proceso de relegamiento científico y de sumisión a las que fueron sometidas (Ortiz, 1996).

\section{ELEMENTOS DEL ARTE DE PARTEAR}

Juan de Navas nunca dirigió la Cátedra de Partos, sino que desde un principio se encargó de la Cátedra de Materia Médica y Fórmulas. Sin embargo, debía poseer amplios conocimientos de obstetricia como lo demuestra el hecho de que en 1795 publicó una obra titulada Elementos del arte de partear. En aquellos momentos los cirujanos no se dedicaban en exclusiva a una especialidad concreta, y la obstetricia no era más que una parte de la cirugía. El mismo Juan de Navas se quejaba en su libro del atraso y la notable decadencia en que se encontraba el arte de partear entre los cirujanos españoles. Achacaba el mayor prestigio de aquellos hombres que practicaban el «arte de partear» en los países donde la obstetricia 
estaba más avanzada, al hecho de que estos se dedicasen en exclusiva a atender partos, a diferencia de los cirujanos españoles «que abrazan al mismo tiempo el vasto campo de la Cirugía» (Navas, 1795).

El objetivo de la obra obstétrica de Juan de Navas era contribuir a paliar la escasez de libros dedicados al «arte de partear» escritos en castellano que sirviesen para instruir a las «comadres». El libro estaba dividido en dos partes, la primera de las cuales, según el propio autor, era la que estaba destinada a las matronas.

\section{LA MUERTE DE JUAN DE NAVAS}

El sueldo que cobraba Juan de Navas, como catedrático, era de dieciocho mil reales de vellón anuales. Al acceder al cargo de vicedirector del Colegio, a finales de marzo de 1798, le fueron concedidos los honores y el sueldo de cirujano de cámara, que ascendía a veinticuatro mil reales de vellón anuales. Sin embargo, no pudo disfrutar demasiado tiempo de dicho aumento de sueldo, porque falleció en la ciudad de Trillo, provincia de Guadalajara, la madrugada del 6 de agosto de 1798 (imagen 1) [1]. Posiblemente Juan de Navas había acudido a Trillo a «tomar las aguas» para recuperarse de alguna dolencia, ya que el balneario disfrutaba de un gran prestigio entre los médicos de la Corte.

A su muerte dejó vacante la Cátedra de Materia Médica y el puesto de vicedirector del Colegio. Manuel Bonafos le sustituyó al frente de la Cátedra de Materia Médica, y Ramón Sarrais fue su sucesor en el puesto de vicedirector del Real Colegio de Cirugía de San Carlos.

Al morir el cabeza de familia, es de suponer que la situación del resto de los miembros empeorase bastante ya que por entonces no eran usuales las pensiones de viudedad u orfandad.
Para ayudar a sus deudos económicamente, la Junta Superior Gubernativa compró a la viuda los apuntes que iban a conformar un libro destinado a su uso en la asignatura de Materia Médica. También revisó la traducción que acababa de completar Juan de Navas, antes de su muerte, de la obra A System of Surgery, del cirujano escocés Benjamín Bell, para que pudiese publicarla, cosa que al parecer no sucedió (imagen 2). Sin embargo, el mismo año de la muerte de Juan de Navas, Santiago García, Académico de número de la Real Academia de Medicina de Madrid y médico de la Real Inclusa, publicó la traducción de ese mismo libro (imagen 3).

Unos meses después de la muerte de Juan de Navas, se aprobó el Reglamento que establecía un Montepío a favor de las viudas, huérfanos y madres de los cirujanos del ejército y de los catedráticos de los Reales Colegios de Cirugía. Posiblemente, el estado de precariedad económica en que había quedado la familia del antiguo vicedirector del Real Colegio de Cirugía de San Carlos alertó al resto de catedráticos, que no quisieron que sus familias sufriesen una situación similar (imagen 4).

\section{CONCEPCIÓN DE NAVAS FERIA}

En noviembre de 1817, con 42 años de edad, Concepción de Navas, hija del «vicedirector que fue» del Real Colegio de Cirugía de San Carlos, solicitó ser admitida en este "para instruirse en el curso de matronas que en él se explica», presentando su instancia en papel timbrado especial para pobres de solemnidad, con un precio de 4 maravedíes. Su marido, Ignacio Dubá, con el que se había casado en Madrid el 11 de mayo de 1803, le firmó la correspondiente autorización que prescribían las Ordenanzas del Real Colegio de Cirugía de San Carlos. El resto de documentos estaba 
formado por su partida de nacimiento y su certificado de matrimonio. Sin embargo solicitaba le fuese dispensada la presentación de los documentos correspondientes a la limpieza de sangre, basándose en que su hermano Juan José de Navas Feria, los había sacado previamente cuando solicitó ingresar en el ejército. Concepción de Navas argumentaba tener que conseguir dicha documentación le sería muy gravoso, empeorando aún más su situación de pobreza, y solicitaba que ya que era de familia conocida le fuera dispensado tal requisito [2].

En su partida de nacimiento consta que Concepción de Navas Feria había nacido el 8 de diciembre de 1774 en Cádiz, y fue bautizada cuatro días más tarde en la Iglesia parroquial castrense del Real Hospital de la Marina. Su madre se llamaba Josefa de Feria Vallejo y era natural de la ciudad de San Lúcar de Barrameda (Cádiz), y su padrino fue José Francisco Sáenz de Santa María. Gracias a los datos obtenidos de esta partida de nacimiento y del certificado de matrimonio, hemos podido completar un pequeño árbol genealógico de la familia (ver imagen 4) [3].

A pesar de no haber completado el tiempo requerido para terminar sus estudios, tras haber «ganado un año literario» Concepción de Navas solicitó poder presentarse a examen para obtener el título de matrona. Dado que había acreditado la práctica particular con un profesor, se aceptó su solicitud mediante una Real Orden de 22 de agosto de 1818 (imagen 6) [4]. Sin embargo, unos años antes, concretamente en 1803, a una antigua alumna del Colegio llamada Rita Sánchez de la Mata Moreda que había solicitado exactamente lo mismo, le fue denegada su petición [5].

Hemos de suponer que, una vez aprobada como matrona, Concepción de Navas, al igual que el resto de las matronas aprobadas por el
Real Colegio de Cirugía de San Carlos, se incorporaría al ejercicio de una profesión bajo unas condiciones muy difíciles, especialmente para aquellas que ejercían dentro de la ciudad de Madrid, caracterizada por los bajos sueldos, las penosas condiciones laborales y dura competencia con los cirujanos de la Corte. Para ilustrar este hecho podemos poner el ejemplo de Juana Pérez Ahumada, matrona examinada y aprobada por tres votos en el Real Colegio de Cirugía de San Carlos de Madrid el 2 de junio de 1830. Juana estaba casada con José Espadín, era de estatura cumplida, tono de piel moreno, ojos y cabello negro y nariz ancha según se recoge en su expediente [6]. Tan solo un año después de obtener su título se desplazó a ejercer a la provincia de Sevilla, donde en su solicitud presentada en el Ayuntamiento para poder ejercer el "arte de la obstetricia», expuso que había tenido que marcharse de la Corte porque allí los cirujanos copaban todo el mercado relacionado con la asistencia al parto (Valle Racero, 1994) [7].

\section{EPÍLOGO}

¿Tendría Concepción de Navas las manos delgadas, los dedos largos y el tacto fino y delicado? ¿Sería perspicaz, ágil y tendría una buena presencia? Todas estas condiciones, además de contar con una buena educación y adecuada conducta cristiana, eran las condiciones necesarias que consideraba su padre que debían tener aquellas personas que se dedicasen a ayudar a las mujeres en el momento del parto (Navas, 1795). Y por último nos preguntamos qué habría opinado Juan de Navas, de haber vivido para verlo, sobre que su hija hubiese continuado con la tradición obstétrica familiar, especialmente si tenemos en cuenta la pobre consideración de que disfrutaban las matronas entre los cirujanos en aquella época. 


\section{NOTAS}

[1]- La documentación relativa a Juan de Navas se encuentra en: Archivo General de la Universidad Complutense de Madrid, AH-0429

[2]- Los datos expuestos se han obtenido de su expediente de alumna en el Colegio de Cirugía de San Carlos, Archivo Histórico Nacional, leg. 1233, exp. 5.

[3]- Hemos localizado en fuentes hemerográficas a un posible hijo de la pareja formada por Ignacio Dubá y Concepción de Navas: se trataría de Miguel Dubá y Navas, nacido en Madrid en 1816 y muerto en Barcelona el 4 de mayo de 1887, escritor y profesor. Pero como no tenemos constatación real de este hecho no le hemos incluido en el árbol genealógico. El árbol genealógico ha sido creado con el software libre disponible en: http://www.myheritage.es/

[4]- Libro copiador de Reales Órdenes del Real Colegio de Medicina y Cirugía de San Carlos, (14 de enero de 1818- 29 de noviembre de 1829). Archivo General de la Universidad Complutense de Madrid, D-0586, folio 27.

[5]- Archivo Histórico Nacional, leg. 1250, exp.4.

[6]- Archivo Histórico Nacional, leg. 1237, exp.158.

[7]- La localización de estos documentos realizada por los investigadores Juan Ignacio Valle Racero y Manuel Jesús García Martínez se ha contrastado solicitando copias al Archivo Municipal de Sevilla. Se encuentran localizados en la sección VI, Libro 56-5, signatura $\mathrm{H} / 1430$.

\section{BIBLIOGRAFÍA}

- Aparicio Simón, J. (1956): Historia del Real Colegio de San Carlos de Madrid. Aguilar, (Madrid).

- Burke, M.E. (1977). The Royal College of San Carlos. Surgery and Spanish Medical Reform in the Late Eighteenth Century. Duke University Press. (Durham).

- Herrera Rodríguez, F. (1989). La aportación de Juan de Navas a la formación de las comadronas (1795). Minutos Menarini, 164, 24-27.

- Navas Ramos, J. (1795). Elementos del arte de partear. Imprenta Real. (Madrid).

- Ortiz Gómez, T. (1996). Género y estrategias profesio- nales. La formación de las matronas en la España del siglo XVIII. En Ramos Palomo, M.D. y Vera Balanza, M.T. (eds.). El trabajo de las mujeres pasado y presente. Actas del Congreso Internacional del Seminario de Estudios Interdisciplinarios de la mujer. Tomo III. Servicio de publicaciones de la Diputación provincial de Málaga, (Málaga), pp. 229-238.

- Real Cédula de S.M. y Señores del Consejo, en que se aprueban y mandan observar las Ordenanzas formadas para el gobierno económico escolástico del Colegio de Círugia establecido en Madrid con el título de San Cárlos. (1787). Imprenta de Pedro Marín, (Madrid).

- Usandizaga, M. (1948). Historia del Real Colegio de Cirugía de San Carlos de Madrid. Consejo Superior de Investigaciones Científicas. (Madrid).

- Valle Racero, J.I. y García Martínez, M.J. (1994) Las matronas en la Historia. Un estudio del siglo XIX. ROL de Enfermería, 187, 61-67.

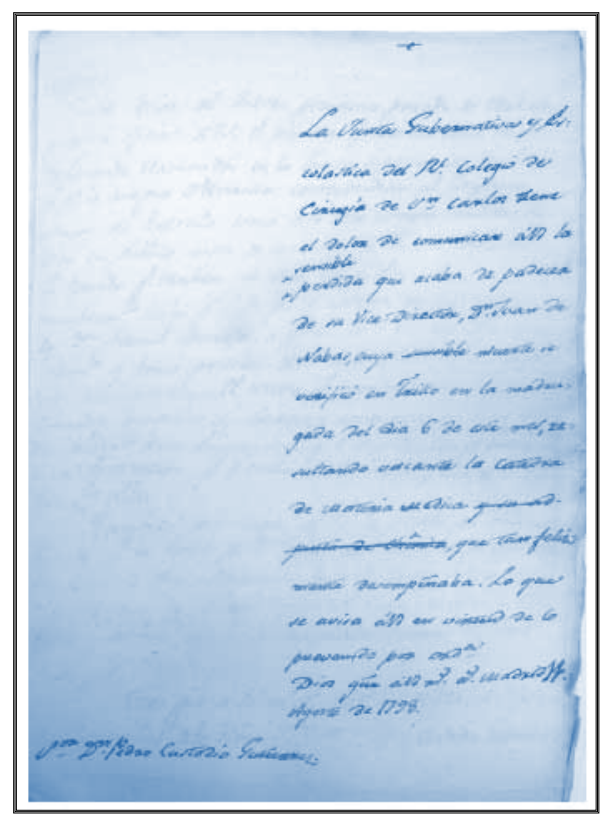

Imagen 1: Comunicación de Pedro Custodio Gutiérrez sobre la muerte de Juan de Navas. 


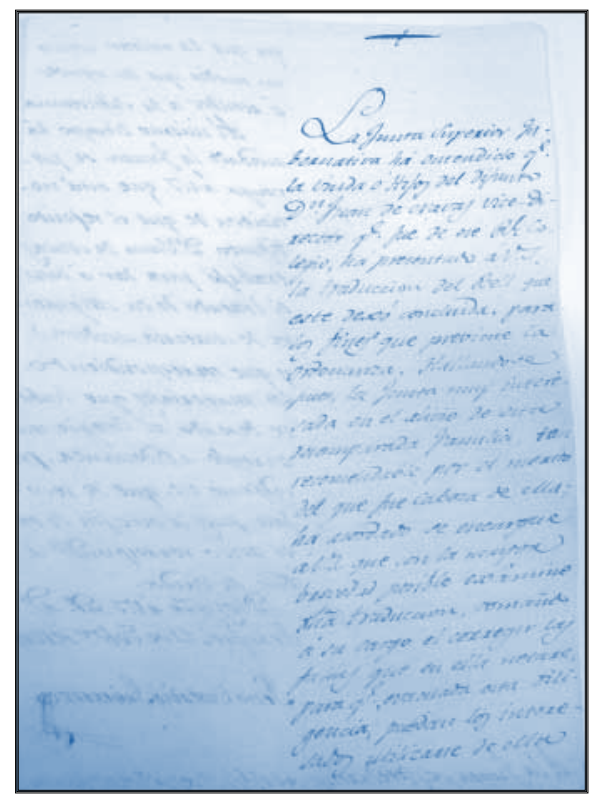

Imagen 2: Preocupación de la Junta Superior Gubernativa por la situación de la familia de Juan de Navas tras su fallecimiento.

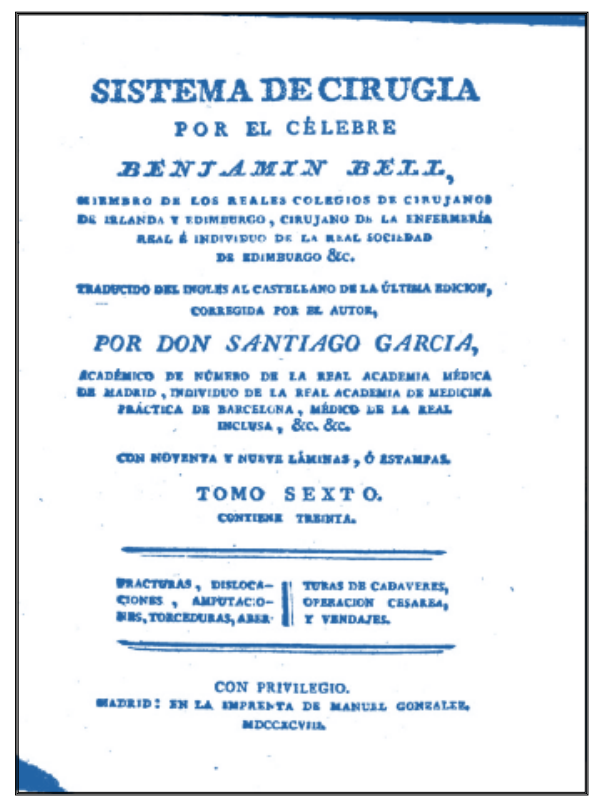

Imagen 3: Portada de la traducción de Santiago García de la obra A System of Surgery de Benjamín Bell.

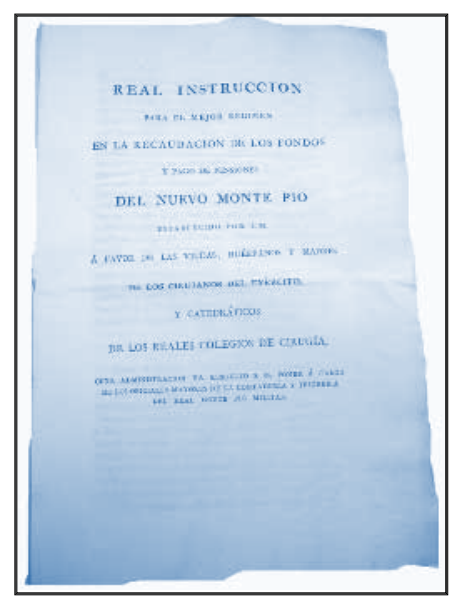

Imagen 4: Montepío a favor de las viudas, huérfanos $y$ madres de los cirujanos del ejército y catedráticos de los Reales Colegios de Cirugía.

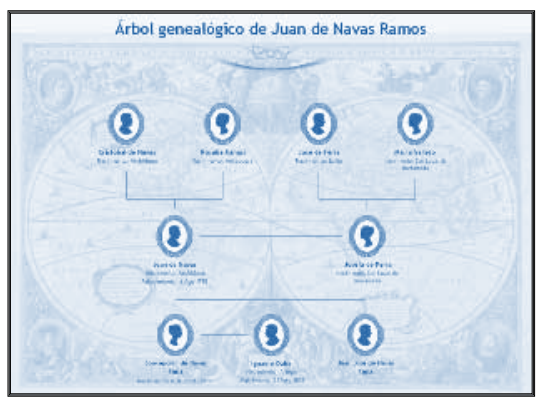

Imagen 5: Árbol genealógico de la familia Navas.

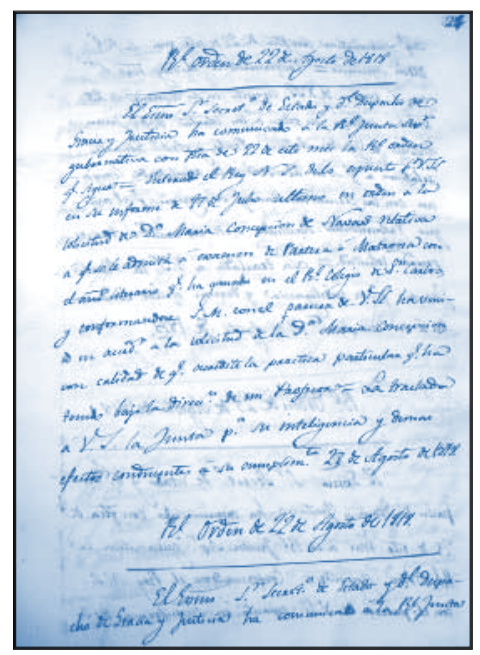

Imagen 6: Real Orden del 22 de agosto de 1818 